\title{
Efectividad en la reducción de la población de Monochamus galloprovincialis (Olivier, 1975; Col.: Cerambycidae) mediante captura masiva.
}

\author{
Sanchez-Husillos, E., ${ }^{1}$ Etxebeste, I., ${ }^{1,2} \&$ Pajares, J. ${ }^{1}$ \\ ${ }^{I}$ Instituto de Investigación Forestal Sostenible, U. Valladolid - CIFOR - INIA. Palencia, España \\ ${ }^{2}$ Departamento de Ingeniería del Medio Agronómico y Forestal, Universidad de Extremadura. ES-10600. \\ *Autor para correspondencia: estela.husillos@gmail.com
}

\section{Resumen}

Monochamus galloprovincialis, vector del nematodo Bursaphelenchus xylophilus, es el causante en Europa de la enfermedad "marchitamiento del pino" (MP).Con su reciente introducción en Europa, la necesidad de medidas de control resulta urgente. La mejora de trampas y atrayentes aumenta las posibilidades de encontrar métodos de reducción de población del vector en zonas afectadas para erradicar la enfermedad o contener su propagación. Para evaluar la eficacia de su captura masiva, se llevaron a cabo dos experimentos de campo. En 2010 se evaluó el método de captura-marcado-recaptura (CMR) para la estimación de la abundancia poblacional, utilizando la formulación POPAN del modelo Jolly-Seber. Las estimaciones derivadas de los parámetros de mejor ajuste demostraron la validez del método. En 2013 se probaron cuatro densidades de trampas en seis parcelas de 36 hectáreas. Para evaluar la proporción de insectos capturados, se estimó en una zona contigua ( $260 \mathrm{ha}$ ) la densidad de la población local del insecto que resultó ser $82 \mathrm{ind} / \mathrm{ha}$. El trampeo extrajo el $4,66 \%, 20.50 \%, 33.33 \%$ y $59.80 \%$ de la población usando $0.02,0.11,0.25$ y 0.44 trampas/ha respectivamente. Una función ajustada con dichos datos muestra que el $95 \%$ de extracción de población se produciría con 0,82 trampas/ha. Estos resultados abren la posibilidad del uso de captura masiva como método de reducción de la población de $M$. galloprovincialis, facilitando así la contención o eventual erradicación de la enfermedad en determinadas zonas afectadas por la enfermedad.

Palabras clave: Bursaphelenchus xylophilus, captura-marcado-recaptura, abundancia, Jolly-Seber, 


\section{Introducción}

Monochamus galloprovincialis (Olivier, 1975), es el vector europeo que transporta el nematodo Bursaphelenchus xylophilus (Steiner \& Bührer, 1934) Nickle, 1970 causante de la enfermedad conocida como marchitamiento del pino (Sousa et al., 2001). Antes de la introducción de la enfermedad en Europa, M. galloprovincialis, fue considerado como un insecto que actuaba como descomponedor primario (Vives, 2000). Sin embargo, la asociación forética obligada del nematodo de la madera del pino (NMP) con el insecto le ha convertido en una especie de prioritaria importancia (Naves et al., 2008). La reciente expansión de la enfermedad en todo Portugal y la detección de cuatro focos en las regiones limítrofes de España (Zamora et al., 2015), ha aumentado la necesidad de desarrollar métodos válidos de contención de la enfermedad.

El desarrollo de trampas con la incorporación de una película de teflón sobre ellas (Rassati et al., 2012; Álvarez et al., 2014), junto con el desarrollo de atrayentes altamente efectivos para la captura de este cerambícido (Álvarez et al., 2016; Ibeas et al., 2007; Pajares et al., 2010)parece ser un factor importante para garantizar el éxito de capturas de Monochamus spp. en grandes cantidades sin opción a escape. Estas mejoras han aumentado la posibilidad de desarrollo de métodos de reducción de la población tales como la captura masiva, lo que puede aumentar la eficacia en los métodos de erradicación o contención de la enfermedad en áreas donde haya sido recientemente introducido. Hasta la fecha, la captura masiva ha sido estudiada como un método de control para plagas de insectos, pero los resultados no siempre han sido convincentes. (Schlyter et al., 2001; El-Sayed et al., 2006; TorresVila et al., 2014). La densidad de población, la movilidad, migración y dispersión del vector son los factores clave que pueden drásticamente reducir el éxito de trampeo masivo. Estudios previos con capturas en trampa de M. galloprovincialis sugieren que este insecto en general se encuentra en bajas densidades (Francardi 2000; Pajares Alonso et al. 2010) pero no existen a día de hoy estimaciones de abundancias absolutas. Sin embargo, para evaluar la efectividad de dicho método es necesario saber previamente la abundancia de la población local. Con este fin se realizaron dos ensayos de campo para evaluar el efecto de la captura masiva sobre poblaciones de M. galloprovincialis y así evaluar si este método es adecuado para reducir la población del vector, para la erradicación o la contención de la enfermedad en las zonas afectadas por la enfermedad.

Nuestros objetivos principales fueron:

- Evaluar un método válido para estimar la abundancia de las poblaciones del insecto, para así poder.

- Comprobar la eficiencia del método de captura masiva para este vector, es decir, la proporción de adultos que podrían ser eliminadas de la población objetivo.

- Estimar la relación entre la densidad de la trampa y la eliminación del vector como ayuda a la toma de decisiones de los gestores fitosanitarios forestales. 


\section{Material y métodos}

\subsection{Estudio preliminar de abundancia de población}

Un primer estudio de captura-marcado-recaptura (CMR) se llevó a cabo para validar este método de estimar la densidad de población de M. galloprovincialis. El estudio se realizó en una masa de Pinus nigra Aiton de 224 ha situado en Villota del Páramo (Palencia) $42^{\circ} 32.026 \mathrm{~N}, 4^{\circ} 51.056 \mathrm{~W}$, del 18 de junio al 30 de septiembre de 2010. Se dispuso de un malla regular con 52 trampas multiembudos cebadas con cebo comercial Galloprotect 2D que se colocaron en los centros de 4 hectáreas (200 x 200 m; 0,25 trampas / ha; Fig. 1). Se colocaron también cuatro trampas adicionales situadas en una malla de 1ha cada celda en las zonas más cercanas al centro, con el objetivo de la detección de individuos que podrían haberse mantenido cerca del punto de liberación.

Se liberaron 250 insectos criados en laboratorio (Tabla 1) en 4 semanas consecutivas en el centro de la malla para ser usados como control, y se llevó a cabo un muestreo semanal de las trampas marcando individualmente y liberando cada insecto entre dos trampas consecutivas.

Tabla 1. Insectos criados en laboratorio liberados en cada ensayo

\begin{tabular}{|l|c|c|c|c|c|c|c|}
\hline Localidad & Año & Ensayo & $\begin{array}{c}\text { Punto } \\
\text { liberación }\end{array}$ & Hembras & Machos & $\begin{array}{c}\text { Peso } \\
\text { medio }( \pm \text { SE })\end{array}$ & $\begin{array}{c}\text { Long } \\
\text { media }( \pm \text { SE })\end{array}$ \\
\hline Cuellar & 2013 & Area población & 7 & 53 & 67 & $199.53 \pm 54.25$ & $12.58 \pm 1.36$ \\
Cuellar & 2013 & Area captura masiva & 7 & 136 & 144 & $216.56 \pm 64.93$ & $12.51 \pm 1.64$ \\
Villota & 2010 & Area población & 4 & 174 & 176 & $417.28 \pm 111.65$ & $15.40 \pm 2.23$ \\
\hline
\end{tabular}

\subsection{Captura masiva}

Un segundo experimento evaluó simultáneamente la densidad de la población nativa y la eliminación de $M$. galloprovincialis utilizando diferentes densidades de trampas dentro de una masa continua de Pinus pinaster Aiton. La zona de estudio se localizó en el municipio de Cuellar ( $41^{\circ} 1707$ N, $4^{\circ} 13022$ W 854 m a. s. 1., Segovia). Se llevaron a cabo ensayos de campo entre el 04 de junio y el 21 de octubre de 2013, cubriendo así todo el período de vuelo del insecto.

El sitio experimental se subdividió en dos zonas contiguas similares de manera que pudo ser evaluado el efecto de las diferentes densidades de trampa en la captura masiva. El sector sur se utilizó para estimar la densidad de población local de $M$. galloprovincialis (Area de Población', fig. 2), mientras que en la zona norte, siete cuadrados (600 x $600 \mathrm{~m})$, con zonas tampón de $100 \mathrm{~m}$ ancho entre ellas se utilizaron para probar cuatro densidades de trampeo ('Área de captura masiva', de 296 ha; Fig.2). Tres subparcelas tenían sólo una trampa en el centro, que corresponde a una densidad de 


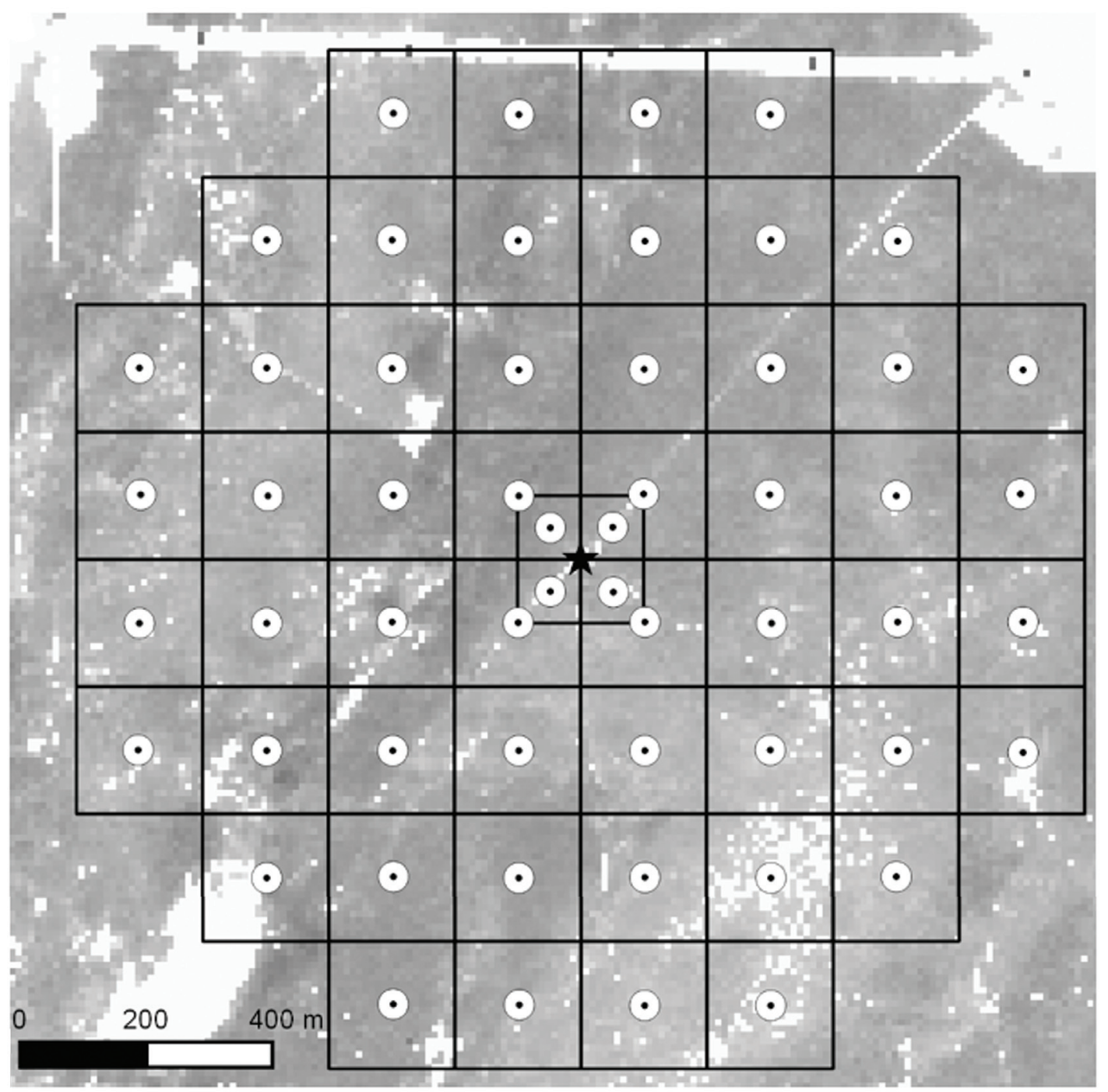

Figura 1. Malla de trampas en ensayo de Villota. Los circulos blancos representan las trampas y la estrella los puntos de liberación.

trampas de 0.02 trampas / ha. En dos subparcelas, se instalaron cuatro trampas siguiendo una cuadrícula regular (300 m distancia entre trampas; 0.11 trampas / ha). Las subparcelas restantes tenían nueve trampas ( $200 \mathrm{~m}$ distancia entre trampas; 0.25 trampas / ha) y 16 trampas en una malla regular (150 m distancia entre trampas; 0.44 trampas / ha), respectivamente (fig 2).

\subsection{Estimación de densidad de población}

En la zona sur se estableció abarcando 290 ha en la que se definió sobre el área una cuadrícula regular de sesenta y cinco cuadrados de 4 hectáreas, de los cuales 20 fueron seleccionados al azar. Una trampa multiembudo cebada se colocó en el cen- 


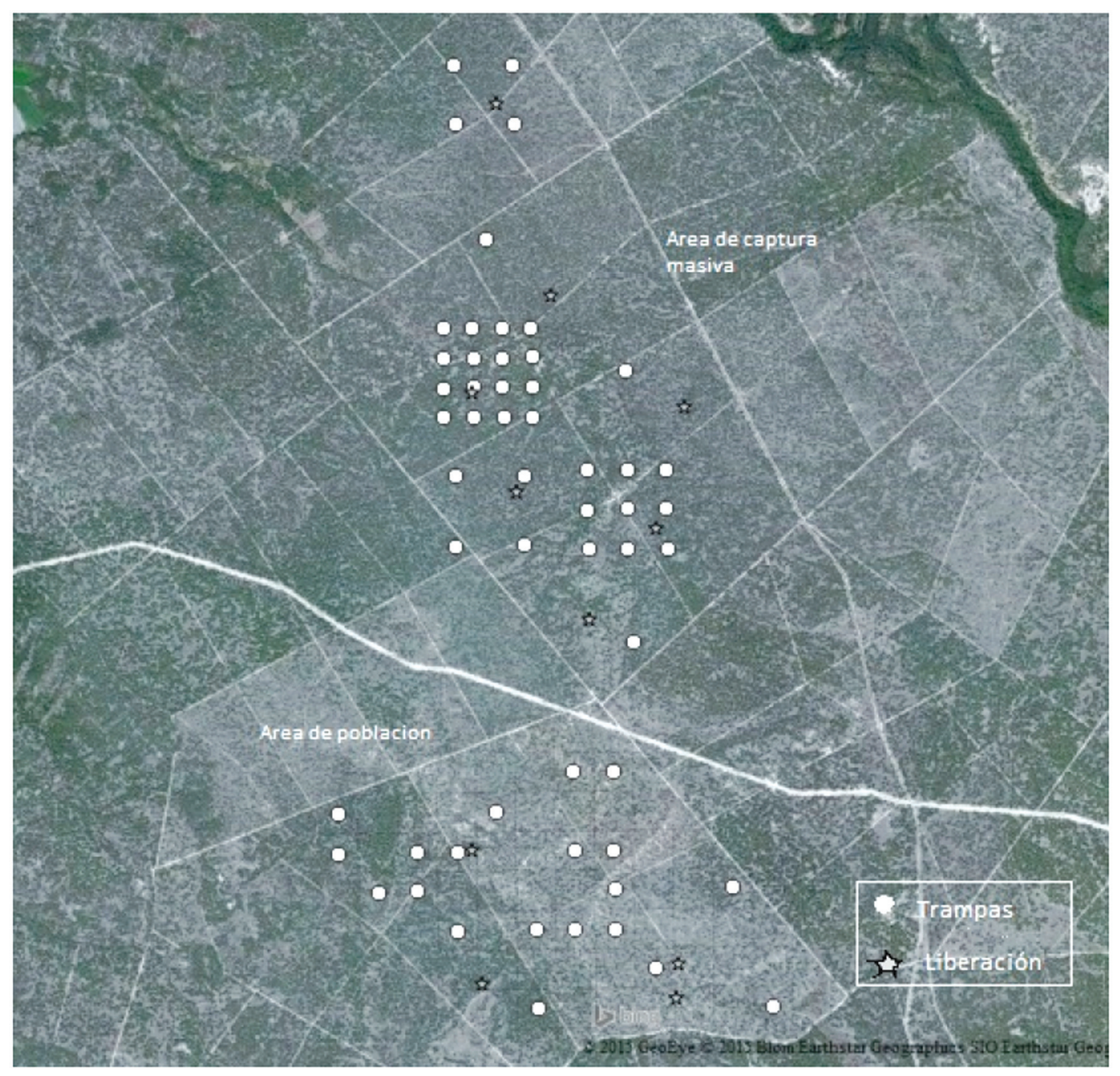

Figura 1. Localización de trampas en ensayo de captura masiva y estima de población en el ensayo de 2013 en Cuellar. Los circulos blancos representan las trampas y las estrellas los puntos de liberación.

tro de cada cuadrado seleccionado (Fig 2). Además, se colocaron ramillos de pino recién cortado dentro del vaso colector de cada trampa para proporcionar alimento y refugio y así aumentar la supervivencia de los insectos atrapados. Todas las trampas fueron revisadas semanalmente y los insectos capturados fueron marcados individualmente y liberados nuevamente entre dos trampas consecutivas. Como en el ensayo de 2010, 399 insectos control criados en laboratorio fueron liberados en 7 puntos aleatorios dentro de la zona de estudio (Tabla 1, figura 2).

\subsection{Análisis estadísticos}

Todos los análisis y cálculos se realizaron mediante el lenguaje de programación de R (Team and R Development Core Team 2010). Análisis demográficos se realiza- 
ron utilizando la biblioteca RMark como interfaz para el paquete MARK, bajo el supuesto de que las poblaciones de M. galloprovincialis durante los periodos de muestreo fueron de tipo abierto, es decir, que se produjeron nacimientos, muertes, emigración e inmigración durante esos períodos. Siguiendo la metodología de Jolly-Seber bajo la parametrización Popan, pudieron obtenerse tres grupos de parámetros primarios: nacimientos $(\mathrm{Bi})$, tamaño de la población $\left(\mathrm{N}_{\mathrm{i}}\right)$ en el intervalo de muestreo y el tamaño total de la población (superpoblación, $\mathrm{N}_{\text {Tot }}$ ). Los parámetros primarios representan: pi, la probabilidad de captura en ocasión i; $\varphi$ i, la probabilidad de sobrevivir un individuo entre ocasiones i y $\mathrm{i}+1$; y bi, la probabilidad de que un insecto de la superpoblación entre en la población entre ocasiones i y i +1 (también conocido como penti o probabilidad de entrada). Estos parámetros primarios se modelan según la notación MARK como constante, $(\bullet)$, dependientes linealmente de tiempo (T), si difieren entre sexos (Sex), o si tienen aditivos o interacciones multiplicativas entre ocasión de muestreo y el sexo (T x Sexo). De este modo se define una gama de modelos para cada parámetro primario. Los mejores modelos fueron seleccionados utilizando el criterio de información de cuasi Akaike (AICC).

Para evaluar el efecto de la captura en la población M. galloprovincialis, es necesario que las estimaciones $\mathrm{N}_{\text {Tot }}$ se transformen en densidad (D). La estimación de D implica asumir algún tipo de cierre geográfica, por lo que se consideró las 290 ha del área definida de estudio. Las capturas acumuladas de cada trampa en la zona de área de captura masiva en 2013 se analizaron de dos formas distintas. En primer lugar, se ajustaron las capturas absolutas por trampa (densidad de trampas) con los factores de los bloques (las subparcelas en las que se colocaron) en un modelo lineal generalizado (GLM), con una distribución de error de Poisson. Las medias fueron posteriormente separadas utilizando la prueba HSD de Tukey con ajuste de Bonferroni. En segundo lugar, las capturas acumuladas por parcela experimental para cada densidad de trampas probada se calcularon. Debido al bajo número de repeticiones, no se aplicaron pruebas paramétricas.

También se realizó una regresión logística para calcular el porcentaje relativo de la población eliminada estimada y la densidad de trampa utilizada cuando se pusieron esos valores en relación con el valor estimado de D. La regresión se ajustó bajo supuestos GLM y una distribución de error quasibinomial para corregir la sobredispersión. Las densidades de trampa en la que el 50\% y el 95\% de la población podría ser extraída (llamadas TD50 y TD95) se computaron sobre el modelo de GLM.

\section{Resultados}

\subsection{Estimación de la abundancia}

El mejor ajuste de los modelos Jolly-Seber (Tabla 2) mostró las probabilidades de supervivencia dependientes del tiempo $(\varphi)$, mientras que la probabilidad de captura (p) o bien se mantuvo constante o dependió del sexo y del tiempo, cuando se permitió la probabilidad de entrada (b) o se fijó a cero. Aunque los tamaños de pobla- 
Tabla 2. Los parámetros del modelo se mantuvieron constantes (.), fijos (por ejemplo 0), difieren entre sexos (Sex), responden al tiempo de forma lineal $(\mathrm{T})$ o puede presentar interacciones (por ejemplo, T x Sex). No. P, significa número de parámetros en el modelo. Las estimaciones derivan de parámetros medios ponderados de los mejores modelos $(\triangle \mathrm{AICc}<10)$. Nliberados es el número de insectos de laboratorio liberados en cada zona de estudio.

\begin{tabular}{|c|c|c|c|c|c|c|}
\hline Insectos & $\mathbf{N}_{\text {liberados }}$ & Mejores modelos & P. $\quad \mathrm{AIC}_{\mathrm{c}}$ & Hembras & Machos & Total \\
\hline \multicolumn{7}{|c|}{ Año 2010} \\
\hline \multirow[t]{2}{*}{$\begin{array}{c}\text { Lab- } \\
\text { liberados }\end{array}$} & $\begin{array}{l}\text { P } 175 \\
\text { ô } 178\end{array}$ & $\left\{p \cdot \varphi \cdot \mathrm{b}_{T \mathrm{x}} \operatorname{Sex} \cdot N.\right\}$ & $8 \quad 217.97$ & $296 \pm 123$ & $277 \pm 127$ & 573 \\
\hline & & $\left\{p_{T \mathrm{x} S e x}, \varphi_{T}, \mathrm{~b}_{\text {fixed }=\mathbf{0}} \cdot N.\right\} 7$ & $7 \quad 230.49$ & $242 \pm 85$ & $216 \pm 85$ & 458 \\
\hline Nativo & & $\left\{p_{T}, \varphi .{ }^{\prime}\right.$ b. $\left.\cdot N.\right\}$ & $5 \quad 109.01$ & & & $115.98 \pm 61.49$ \\
\hline \multicolumn{7}{|c|}{ Año 2013} \\
\hline $\begin{array}{l}\text { Lab- } \\
\text { liberados }\end{array}$ & $\begin{array}{l}\text { o } 188 \\
\text { ô } 211\end{array}$ & $\left\{p_{T \mathrm{x} S e x}, \varphi_{T}, \mathrm{~b}_{\text {fixed }}=\mathbf{0}\right.$ & 9135.66 & $143 \pm 151$ & $132 \pm 151$ & 275 \\
\hline Nativo & & $\left\{p_{T \mathrm{x} S e x}, \varphi_{T}, \mathrm{~b}_{T} \cdot N.\right\}$ & 92468.38 & $10835 \pm 1289$ & $10484 \pm 1282$ & 21319 \\
\hline
\end{tabular}

ción derivados bajo ambos escenarios sobreestimaron las cifras reales, cayeron dentro de un error estándar de 175 hembras liberadas y 178 machos (tabla 1), y por lo tanto la precisión de las estimaciones de abundancia derivados de los datos de CMR fue aceptada como correcta.

El modelo Jolly-Seber que mejor se ajusta reveló que la supervivencia y probabilidades de entrada para nativos en 2013 eran dependientes del tiempo (Tabla 2). También pudo derivarse una interacción tiempo-sexo en la probabilidad de captura. El promedio ponderado de modelos bajo la parametrización de Popan dio lugar a la estimación de una superpoblación de 21319 individuos de M. galloprovincialis. Sin embargo, las estimaciones semanales oscilaron entre 291 y 1138 insectos (Fig. 3). Considerado el área, la densidad de la población resultó 82 individuos/ha.

\subsection{Efecto de la captura masiva}

Se capturaron un total de 5113 insectos nativos durante todo el período de captura en la zona de captura masiva de 2013.

Las estimaciones de abundancia semanales fueron transformadas a densidades para calcular los porcentajes de eliminación de la población durante el período de muestreo. Aunque el valor más alto por trampa fue obtenido con 0.11 trampas ha- 1 (177.25 \pm 11.84 SEM de M. galloprovincialis por trampa) (Fig. 4), la parcela con densidad de trampas de 0.44 ha-1 capturó un total de 2065 individuos (129.06 \pm 8.01 SEM individuos por trampa), proporcionando la más alta captura por unidad de área (57.36 insectos ha-1; Fig. 5). 


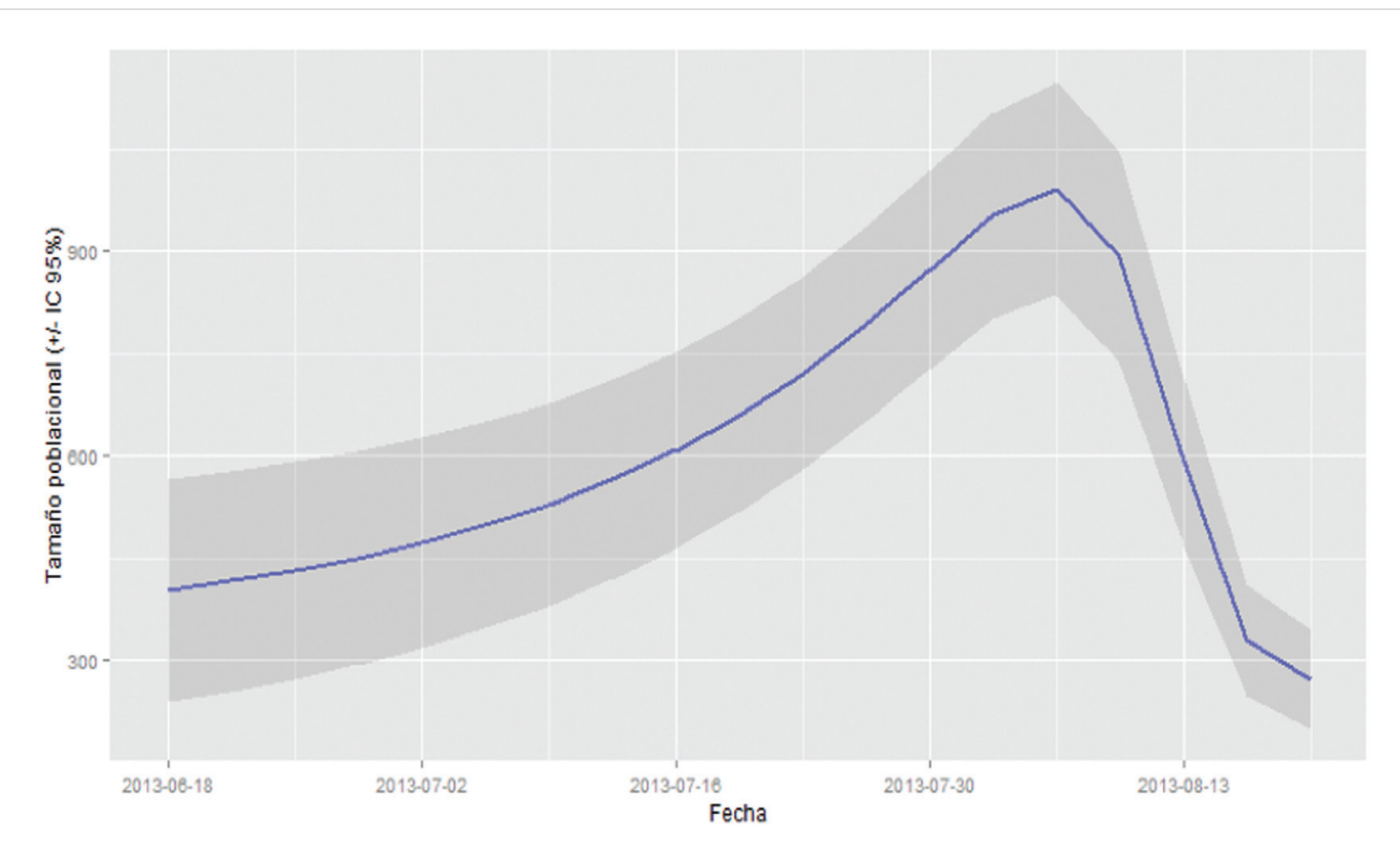

Figura 3. Abundancias de población de Monochamus galloprovincialis estimadas por semana en el ensayo de 2013 de Cuellar.

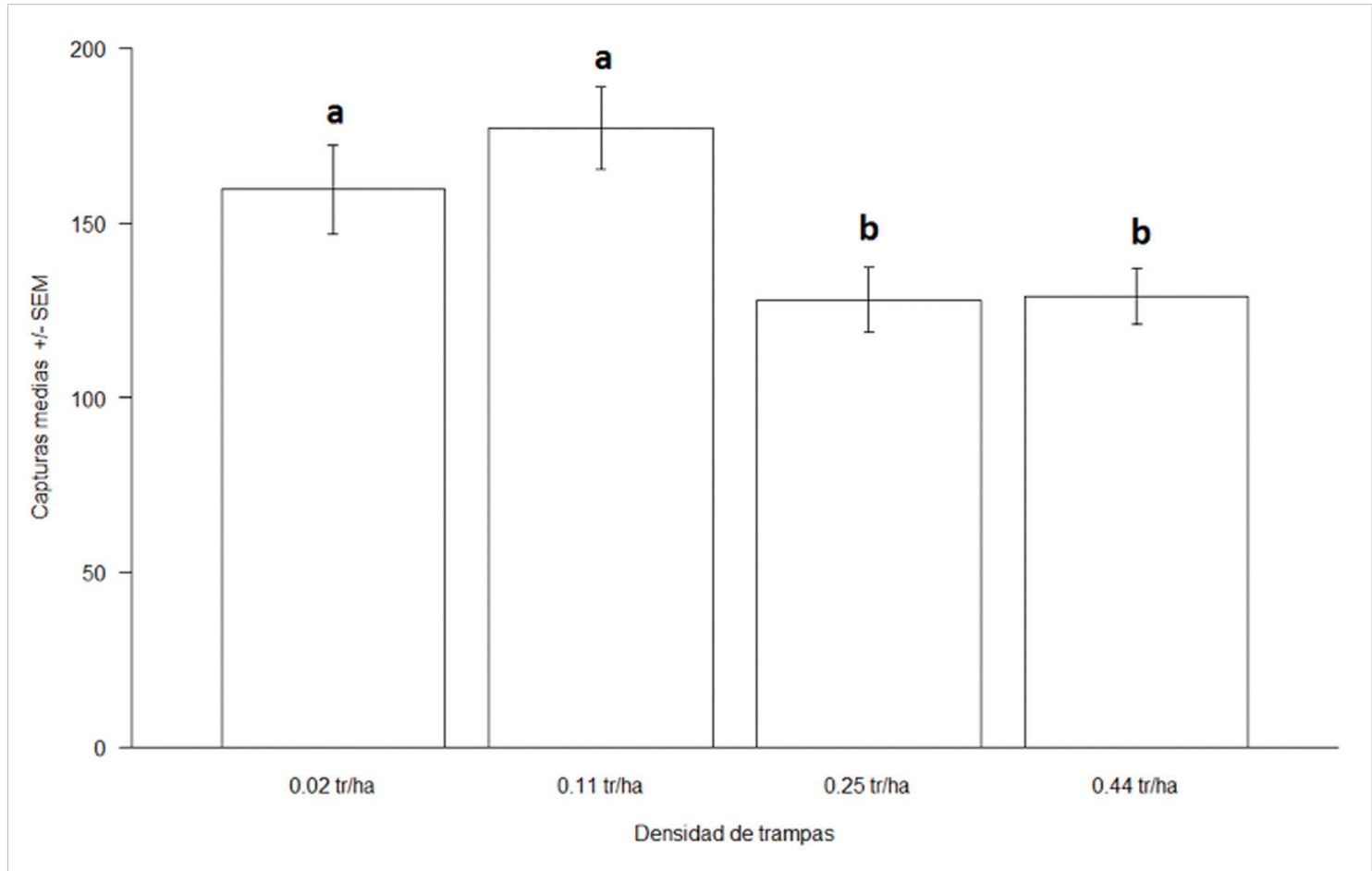

Figura 4. Capturas por trampa de Monochamus galloprovincialis \pm SEM para las densidades de trampas en Cuéllar durante el ensayo de 2013. Barras que comparten la misma letra no son significativamente diferentes (Tukey HSD, ajuste de Bonferroni, $\mathrm{P}<0,05$ ). 


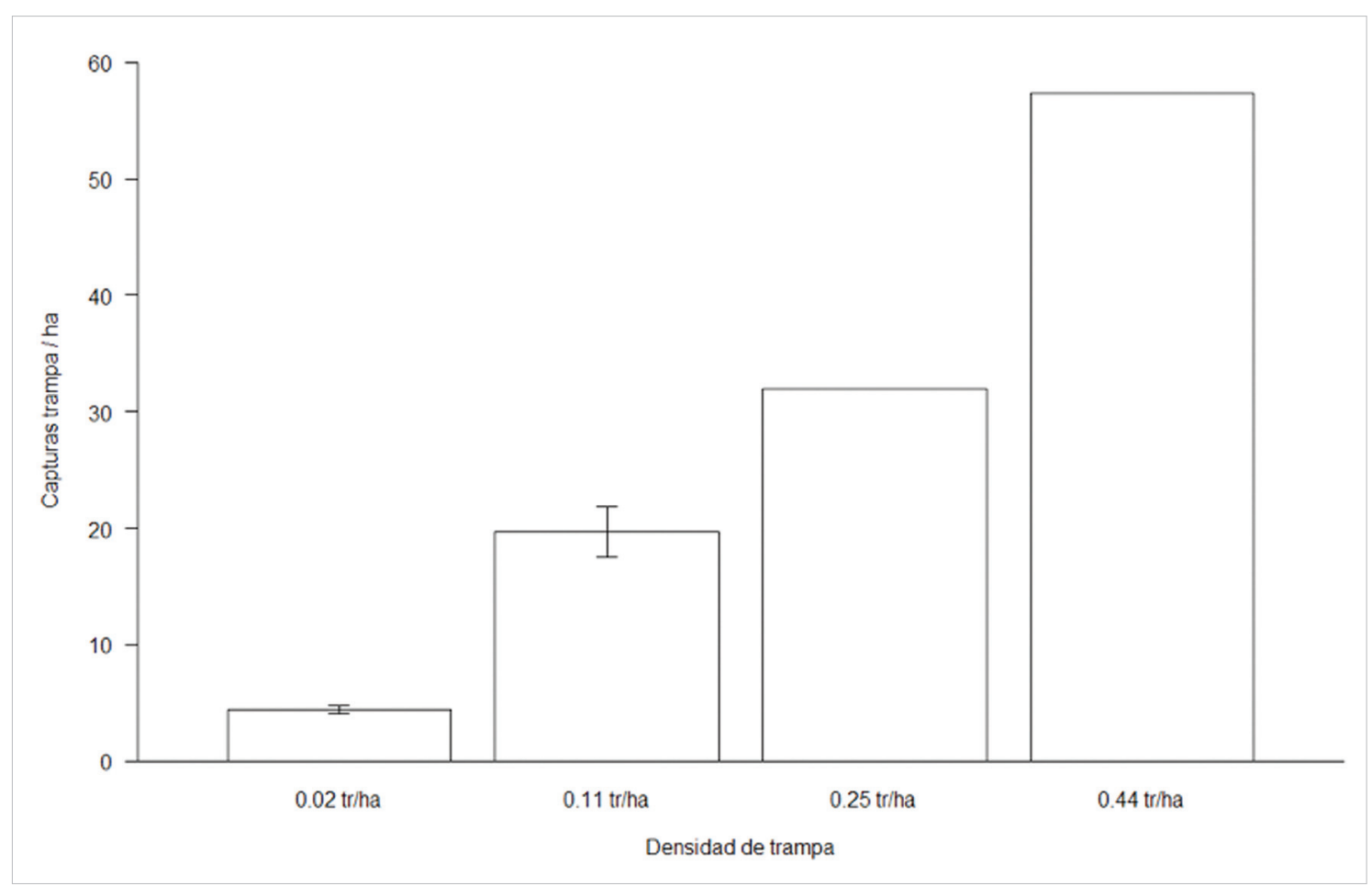

Figura 5. Capturas totales de Monochamus galloprovincialis \pm SEM para las densidades de trampas en Cuéllar durante el ensayo de 2013.

El modelo para calcular la densidad de trampas óptima se realizó utilizando el valor estimado de 82 individuos ha-1 de densidad de insectos nativos. La mayor densidad de trampas mayor (0.44 tramps/ha) retiró el $59.80 \%$ de la población y la densidad de trampas menor (de 0.02 trampas/ha) retiró sólo el $4.66 \%$ de la población. El modelo logístico predijo que los valores de las densidades de trampas requeridas para retirar el $50 \%$ de la población resultó ser $0.37 \pm 0.04 \mathrm{SE}$ trampas ha-1 y para extraer el $95 \%$ podrían ser densidades cercanas al $0.82 \pm 0.11$ SE trampas ha- 1 respectivamente, aunque este dato está por encima del rango modelizado.

\section{Discusión}

Las estimaciones de abundancia de la población usada como control derivadas de los datos de CMR de M. galloprovincialis eran bastante consistentes con los verdaderos valores (Tabla 2), lo que indica que las abundancias estimadas para los nativos también se acercarían a sus verdaderos valores. En todo caso, los valores en 2010 indicaron abundancias ligeramente sobreestimadas.

El aumento de las densidades de trampas conlleva un aumento de las capturas por parcela (Fig. 4 y Fig. 5). Así, las parcelas con la más alta densidad de trampas (0.44 trampas ha-1) fueron capaces de capturar 57.36 insectos ha-1 (2065 dentro de la par- 
cela de 36 hectáreas), o $59.80 \%$ de la población residente estimada. Si bien las extracciones superiores al 59.8\% se encuentran fuera del rango del modelo y por lo tanto sometidas a una gran incertidumbre, es posible que densidades superiores de trampas de 1.5 trampa/ha podrían eliminar casi el $100 \%$ de la población. Sin embargo, utilizando sólo la mitad de las trampas (0.82 trampas ha-1) podría dar lugar a una reducción del 95\% de la densidad de población.

En circunstancias normales, los Monochamus son insectos secundarios que se reproducen en árboles recién muertos o moribundos y ramas. La disponibilidad de este tipo de material hospedante depende de las condiciones del bosque que pueden variar fuertemente espacial y temporalmente (Grove, 2002). Bajo tal supuesto, se espera que las poblaciones de $M$. galloprovincialis existan principalmente en bajas densidades. Sin embargo, de acuerdo con nuestros resultados, las densidades de insectos nativos pueden variar fuertemente $(0.52$ y 82 individuos ha- 1 en 2010 y 2013 respectivamente). La proporción de insectos eliminados sugiere que la captura masiva puede ser aplicada operativamente incluso en poblaciones con densidades aparentemente moderadas. Los niveles de población reportados para M. alternatus Hope 1842 (Shibata ,1985; Togashi, 1988) conducen a preguntarse sobre los niveles poblacionales de $M$. galloprovincialis que pueden alcanzarse en zonas afectadas por la enfermedad, y si la captura masiva podría ser útil como método de control con estos niveles. Esto implica la necesidad de seguir ampliando el conocimiento de las dinámicas poblacionales de estos insectos y de la eficiencia de la captura masiva en la extracción de la población a elevadas densidades poblacionales, como la que cabría esperar por ejemplo en un escenario de brote de la enfermedad en la que se van a originar grandes cantidades de material colonizable para el insecto.

De acuerdo con nuestros resultados, el trampeo masivo puede ayudar efectivamente a detener la propagación de la enfermedad del marchitamiento del pino, tanto en programas de erradicación en las zonas donde la enfermedad se ha detectado recientemente como en las zonas fronterizas u otras zonas tampón donde la contención es fuertemente necesaria.

\section{Conclusiones}

La formulación de POPAN usando el método de captura-marcado-recaptura es un método válido para la estimación de abundancias poblacionales de Monochamus galloprovincialis en las localidades estudiadas.

El método de captura masiva es eficaz con este insecto dando como resultado que el $60 \%$ de extracción de población se produciría con una densidad de 0,44 trampas/ha.

Estos resultados sugieren que la reducción sustancial de la abundancia de $M . g a$ lloprovincialis podría lograrse a través de la captura masiva y que esto representa un método de gestión muy prometedor para ayudar a la contención o erradicación futura de B. xylophilus en zonas fronterizas o de bordes afectadas por la enfermedad donde la contención es especialmente importante. 


\section{Agradecimientos}

Nos gustaría agradecer la asistencia de campo proporcionado por G. Álvarez y A. Ponce, y por el equipo del Centro de Sanidad Forestal Calabazanos, de Castilla y León, que nos ayudó en la cría de los insectos, y a los agentes forestales de la Junta de Castilla y León del Servicio Forestal en Segovia por el acceso a los sitios de campo. Este trabajo ha sido financiado a través del proyecto de la Unión Europea REPHRAME (FP7-KBBE-2010-4) y por el Ministerio de Ciencia español mediante el proyecto de innovación (RTA2011-00069-C03-03). I. Etxebeste fue parcialmente financiado por la subvención POSDOC del Gobierno Autónomo Vasco.

\section{Bibliografía}

Álvarez, G., Etxebeste, I., Gallego, D., David, G., Bonifacio, L., Jactel, H., Sousa, E., Pajares,J., 2014. Optimization of Traps for Live Trapping of Pine Wood Nematode Vector Monochamus galloprovincialis. Journal of Applied Entomology 139 (8): n/a - n/a. doi:10.1111/jen.12186.

Álvarez, G.,Gallego, D.,David, R., Hall, D.,Jactel, H ., Pajares, J., 2016. Combining Pheromone and Kairomones for Effective Trapping of the Pine Sawyer Beetle Monochamus galloprovincialis. Journal of Applied Entomology. 140(1-2) 58-71.doi: 10.1111/ jen.12297.

El-Sayed, A M., Suckling, D M., Wearing, C H., J A Byers., 2006. Potential of Mass Trapping for Long-Term Pest Management and Eradication of Invasive Species. Journal of Economic Entomology 99 (5): 1550-64. doi:10.1603/0022-0493-99.5.1550.

Francardi, V., 2000. Distribution and Abundance of Monochamus Species in Italy. In XXI International Congress of Entomology, 484.

Grove, SJ., 2002. Saproxylic insect ecology and the sustainable management of forests. Annu Rev Ecol Syst 33,1-23.doi: 10.1146/annurev.ecolsys.33.010802.150507

Ibeas, F., Gallego, D., Díez, J., y Pajares J.A., 2007. An Operative Kairomonal Lure for Managing Pine Sawyer Beetle Monochamus Galloprovincialis (Coleoptera: Cerymbycidae). Journal of Applied Entomology 131 (1): 13-20. doi:10.1111/j.1439-0418. 2006.01087.x.

Naves, P., Sousa,E., Rodrigues,JM., 2008. Biology of Monochamus galloprovincialis (Coleoptera, Cerambycidae ) in the Pine Wilt Disease Affected Zone, Southern Portugal. Silva Lusitana 16 (2). Unidade de Silvicultura e Produtos Florestais: 133-48.

Pajares, J.A., Álvarez,G., Ibeas,F., Gallego,D., Hall,D., y Farman D., 2010. Identification and Field Activity of a Male-Produced Aggregation Pheromone in the Pine Sawyer Beetle, Monochamus galloprovincialis. Journal of Chemical Ecology 36 (6): 570-83. doi:10.1007/s10886-010-9791-5.

Rassati, D., Petrucco Toffolo, E., Battisti, A., Faccoli. M.,2012. Monitoring of the Pine Sawyer Beetle Monochamus galloprovincialis by Pheromone Traps in Italy. Phytoparasitica 40 (4): 329-36. doi:10.1007/s12600-012-0233-5.

Schlyter,H., Fredrik, B., Zhang, QH., Liu, GT., Ji. LZ.,2001. A Successful Case of Pheromone Mass Trapping of the Bark Beetle Ips Duplicatus in a Forest Island, Analysed 
by 20-Year Time-Series Data. Integrated Pest Management, Weslien 1992: 185-96. doi: 10.1023/A:1025767217376

Shibata, E., 1985. Seasonal fluctuation of the pine woodnematode, Bursaphelenchus xylophilus (Steiner et Buhrer)Nickel (Nematoda, Aphelenchoididae), transmitted to pine by the Japanese pine sawyer, Monochamus alternatus Hope (Coleoptera, Cerambycidae). Appl Entomol Zool 20, 241-245.doi: 10.1303/aez.20.241

Sousa, E., Bravo, MA., Pires, J., y Naves. P.,2001. Bursaphelenchus Xylophilus (Nematoda; Aphelenchoididae) Associated with Monochamus Galloprovincialis (Coleoptera; Cerambycidae) in Portugal. Nematology 3: 89-91. doi: 10.1163/156854101300106937

Team, R Development Core, and R R Development Core Team., 2010. R: A Language and Environment for Statistical Computing. Edited by R Development Core Team. R Foundation for Statistical Computing. Vol. 1. R Foundation for Statistical Computing. doi:10.1007/978-3-540-74686-7.

Torres-Vila, L.,Zugasti, MC., De-Juan, J. M., Oliva, M. J., Montero, C., Mendiola, F. J., Conejo, Y.,. 2014. Mark-Recapture of Monochamus galloprovincialis with Semiochemical-Baited Traps: Population Density, Attraction Distance, Flight Behaviour and Mass Trapping Efficiency. Forestry 88 (2): 224-36. doi:10.1093/forestry/cpu049.

Togashi, K., 1988. Population-density of Monochamus alternatus adults (Coleoptera, Cerambycidae) and incidence of pine wilt disease caused by Bursaphelenchus xylophilus (Nematoda, Aphelenchoididae). Res Popul Ecol (Kyoto) 30, 177-192.doi: 10.1007/BF025 13243

Vives, E., 2000. Insecta Coleoptera Cerambycidae. In Fauna Ibérica, 2nd ed., 12:vol 12. Madrid: CSIC (Ed.), Fauna Ibérica. Museo Nacional de ciencias Naturales.

Zamora, P., Rodríguez, V., Renedo, F., Sanz, AV., Domínguez, JC., Escolar, G., Miranda, J.,Navas,A.,Robertson, L.,Martin, AB., 2015. First Report of Bursaphelenchus Xylophilus Causing Pine Wilt Disease on Pinus Radiata in Spain | International Biosecurity Intelligence System. Plant Disease. 99:10,1449-1449 